\title{
CRISPR/Cas9 gene-editing platform corrects mutations associated with Duchenne muscular dystrophy
}

Gene editing via the CRISPR/Cas9 system can restore dystrophin expression in myoblasts from patients with Duchenne muscular dystrophy (DMD), according to a new report in Nature Communications.

$\mathrm{DMD}$ is caused by mutations in the dystrophin gene, most of which are frameshift deletions. "The unique

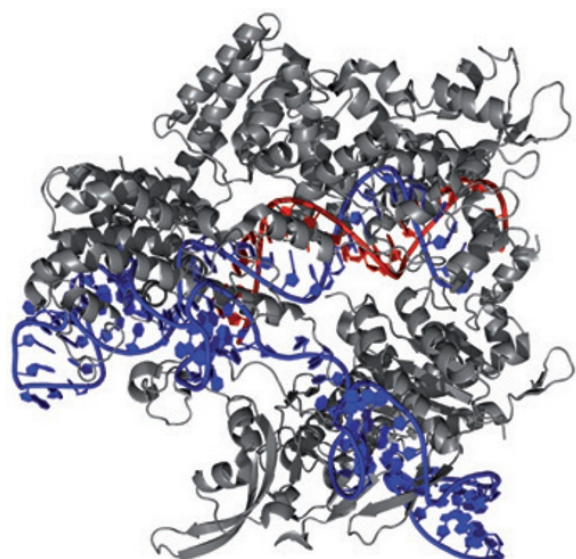

The crystal structure of the CRISPR/Cas9 complex with DNA. Image courtesy of C. A. Gersbach. molecular basis of dystrophin mutations that cause DMD is well suited for genome editing, since function can be restored by simply removing gene segments without needing to perfectly restore the complete gene," explains Charles Gersbach, who led the new study.

The CRISPR/Cas9 system, which was adapted from a microbial system that confers resistance against phage invasion, uses single guide RNAs (sgRNAs) to direct the Cas9 nuclease to predefined sites within a gene. Gersbach's team designed a range of sgRNAs to target various sites in the exon 45-55 region of the dystrophin gene-a 'hot spot' that harbours more than $60 \%$ of known DMD-causing mutations.

The researchers used the CRISPR/Cas9 system to excise the entire dystrophin exon 45-55 region in myoblasts from patients with DMD. This intervention restored the reading frame of the gene and enabled the cells to produce a shortened but functional form of the protein.
"The demonstration that dystrophin can be restored by simply delivering a single Cas9 enzyme and two small RNAs is a major advance in designing gene-editing strategies that are relevant to over $60 \%$ of DMD patients," concludes Gersbach. "Previous approaches required geneediting reagents for specific mutations that vary between patients-a much more challenging drug development path.”

Future research will address whether the CRISPR/Cas9 approach can ameliorate the symptoms of DMD in animal models and, ultimately, humans. In addition, Gersbach and colleagues intend to explore the possibility of delivering the CRISPR/ Cas9 system directly to skeletal and cardiac muscle in vivo.

Heather Wood

Original article Ousterout, D. G. et al. Multiplex CRISPR/ Cas9-based genome editing for correction of dystrophin mutations that cause Duchenne muscular dystrophy. Nat. Commun. 6, 6244 (2015) 